\title{
Editorial
}

\section{Operator Methods in Approximation Theory}

\author{
${ }^{1}$ Department of Mathematics, Computer Science and Economics, University of Basilicata, Potenza, Italy \\ ${ }^{2}$ Department of Mathematics, Faculty of Science, Selcuk University, Selcuklu, Konya, Turkey \\ ${ }^{3}$ Department of Mathematics, University of Bari, Bari, Italy \\ ${ }^{4}$ Department of Mathematics, University of Jaén, Jaén, Spain
}

Vita Leonessa $\mathbb{D}^{\mathbb{D}},{ }^{1}$ Tuncer Acar $\mathbb{D}{ }^{2},{ }^{2}$ Mirella Cappelletti Montano, ${ }^{3}$ and Pedro Garrancho $\mathbb{D}^{4}$

Correspondence should be addressed to Vita Leonessa; vita.leonessa@unibas.it

Received 16 June 2019; Accepted 16 June 2019; Published 11 July 2019

Copyright (C) 2019 Vita Leonessa et al. This is an open access article distributed under the Creative Commons Attribution License, which permits unrestricted use, distribution, and reproduction in any medium, provided the original work is properly cited.

Approximation theory is an intensive research area, developed in different directions by many mathematicians.

For example, approximation and iteration processes arise in a very natural way in many problems dealing with the constructive approximation of functions as well as solutions to (partial) differential equations and integral equations. Moreover, approximation theory can be successfully applied in fixed point theory, in computer aided geometric design, in artificial neural networks, in the study of evolution problems, and in function algebras.

The goal of this special issue is to attract original research and review articles that highlight recent advances in operator methods within approximation theory and related applications.

The interest aroused by the mathematicians who work in this area is remarkable, as evidenced by the thirty-six submissions received.

The papers that have been accepted for the publication in the issue recover the following topics: means and inequalities, approximation by positive operators, function algebras, fixed point theorems, and iteration processes.

In what follows we give a brief description of the contents of this special issue.

In the review article titled "On Sequences of J. P. KingType Operators," T. Acar et al. provide an essential exposition of a series of investigations developed in the last fifteen years after the release of a paper written by J. P. King, where a modification of the classical Bernstein operators was considered in order to get better approximation properties than the original ones. After a brief history devoted to the sequences of positive linear operators fixing certain (polynomial, exponential, or more general) functions obtained by applying King's approach, the authors illustrate certain Kingtype modifications of the well-known Bernstein and SzászMirakjan operators.

A. A. Bakery and M. M. Mohammed in the paper "Small Pre-Quasi Banach Operator Ideals of Type Orlicz-Cesáro Mean Sequence Spaces" deal with determining sufficient conditions on an Orlicz-Cesàro mean sequence space ces in order that the class $S_{c e s_{\varphi}}$, consisting of all bounded linear operators between arbitrary Banach spaces such that the corresponding sequence of $s$-numbers belongs to $c e s_{\varphi}$, forms an operator ideal. Moreover, the authors determine some inclusion relations between pre-quasi operator ideals as well as their duals. Finally, they present sufficient conditions on $\operatorname{ces}_{\varphi}$ in order that the pre-quasi Banach operator ideal generated by approximation number is small.

The purpose of the paper "Asymptotic Behavior of Almost Quartic *-Derivations on Banach *-Algebras" by H.M. Kim et al. is determining, in the context of Banach *algebras, stability theorems of quartic $*$-derivations associated with the quartic functional equation $f(3 x-y)+f(x+y)$ $+6 f(x-y)=4 f(2 x-y)+4 f(y)+24 f(x)$.

S. Z. Ullah et al. generalize and improve some known results concerning integral majorization type and generalized Favard's inequalities for the class of strongly convex functions in the paper titled "Integral Majorization Type Inequalities for the Functions in the Sense of Strong Convexity."

In the paper "Bivariate Chlodowsky-Stancu Variant of (p, q)-Bernstein-Schurer Operators” by T. Vedi-Dilek and E. 
Gemikonakli, the bivariate Chlodowsky-Stancu variant of $(p, q)$-Bernstein-Schurer Operators, as well as a generalization of it, is proposed and some approximation properties are investigated. The paper ends by discussing some numerical results.

N. Özmen in the paper titled "New Generating Function Relations for the q-Generalized Cesàro Polynomials" examines a $q$-analogue of generalized Cesàro polynomials for which she derives bilinear and bilateral generating functions; in addition, she gets a specific linear $q$-generating relationship that recovers the basic analogue of certain special functions.

H. J. Lee proves that the $k$-homogeneous polynomial and analytic numerical index of certain $X$-valued function algebras, $X$ being a complex Banach space, are the same as those of $X$ in the paper titled "Generalized Numerical Index of Function."

In the paper "Convergence Analysis of an Accelerated Iteration for Monotone Generalized $\alpha$-Nonexpansive Mappings with a Partial Order," a new accelerated iteration process for finding fixed points of monotone generalized $\alpha$ nonexpansive mappings in ordered Banach spaces is introduced. Y.-A. Chen and D.-J. Wen establish some weak and strong convergence theorems of fixed point for monotone generalized $\alpha$-nonexpansive mappings in a uniformly convex partially ordered Banach space. Moreover, they provide a numerical example that illustrates the convergence behavior and effectiveness of their method.

C. Zhang and S. Wang in the paper titled "Structure Properties for Binomial Operators" discussed some structures properties of the binomial operators, such as moments representation, derivatives representation, and binary representation. As applications, the authors prove that the binomial operators considered preserve increasing functions, convex functions, and Hölder (continuous) functions.

The purpose of the paper "On New Picard-Mann Iterative Approximations with Mixed Errors for Implicit Midpoint Rule and Applications" written by T. Li and H. Lan is to introduce and study a new class of Picard-Mann iteration processes with mixed errors for the implicit midpoint rules and to analyze the convergence and stability of the proposed method. Some numerical examples and applications to optimal control problems with elliptic boundary value constraints are presented, and they show that the Picard-Mann iteration process discussed in the article is more effective than other related iterative processes.

J.-L. Wang et al. in the paper titled "On Approximating the Toader Mean by Other Bivariate Means" provide several sharp bounds for the Toader mean by using certain combinations of the arithmetic, quadratic, contraharmonic, and Gaussian arithmetic geometric means.

The paper " $C^{*}$-Basic Construction from the Conditional Expectation on the Drinfeld Double" by Q. Xin et al. deals with the following topics. Let $G$ be a finite group and $H$ a subgroup of $G$. Starting from the Drinfeld double $D(G)$ and the crossed product $D(G ; H)$ of $C(G)$ and $C H$, and considering the $C^{*}$-basic construction $C^{*} \mathrm{fbffk} D(G)$, efbfft from the conditional expectation $E$ of $D(G)$ onto $D(G ; H)$, the authors construct a crossed product $C^{*}$-algebra $C(G / H \times$
$G) \rtimes \mathrm{CG}$, in such a way that $C^{*} \mathrm{fbffk} D(G)$, efbfft is $C^{*}$-algebra isomorphic to $C(G / H \times G) \rtimes C G$.

In the paper "On a New Stability Problem of Radical $n$ th-Degree Functional Equation by Brzdęk's Fixed-Point Method," D. Kang and H. B. Kim, given a positive integer $n$, discuss the general solutions to the radical $n$-th degree functional equation $f\left(\sqrt[n]{x^{n}+y^{n}}\right)=f(x)+f(y)$ and prove new Hyers-Ulam type stability results by using Brzdęk's fixed point method.

In the context of singular Hadamard fractional boundary value problems, J. Mao et al. establish, by using an iterative algorithm, the existence and uniqueness of the exact iterative solution in the paper titled "The Unique Positive Solution for Singular Hadamard Fractional Boundary Value Problems." Moreover, they show the iterative sequences converge uniformly to the exact solution, and they provide estimation of the approximation error and the convergence rate.

\section{Conflicts of Interest}

The Guest Editors declare that they have no conflicts of interest regarding the publication of this special issue.

\section{Acknowledgments}

We are very grateful to the mathematical community for the great interest shown in this special issue. In particular, we want to thank all the authors of the published papers for their contribution in this field. Also, our gratitude goes to the reviewers, for their precious help in handling all the submitted manuscripts, and to Hindawi who supported us throughout the development of this special issue. Finally, the Lead Guest Editor sincerely thanks her Guest Editors for agreeing to join her in this project.

Vita Leonessa Tuncer Acar Mirella Cappelletti Montano Pedro Garrancho 


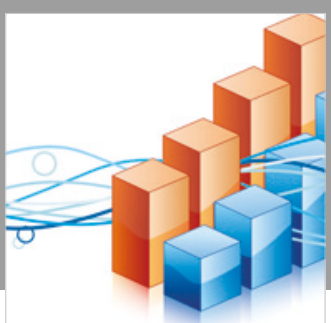

Advances in

Operations Research

\section{-n-m}
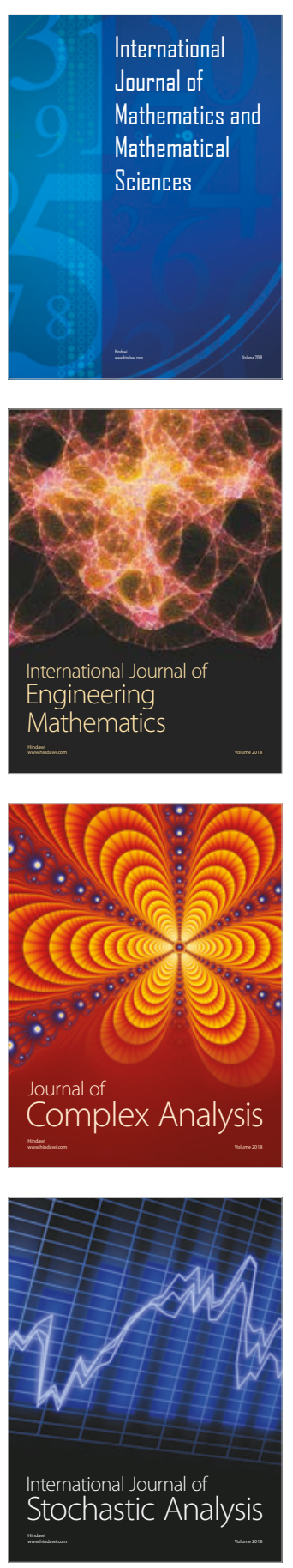
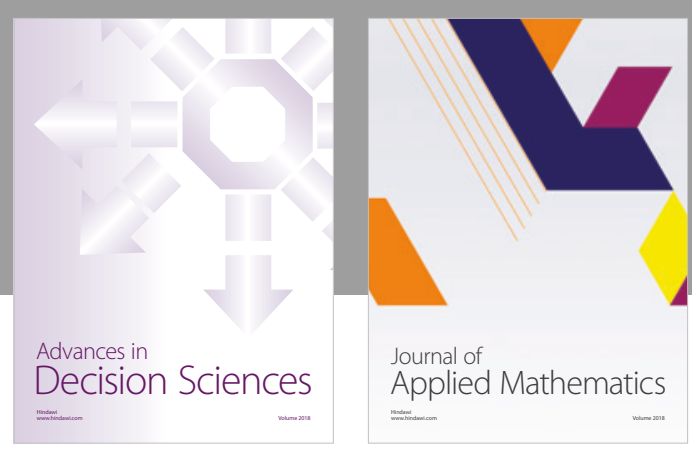

Journal of

Applied Mathematics
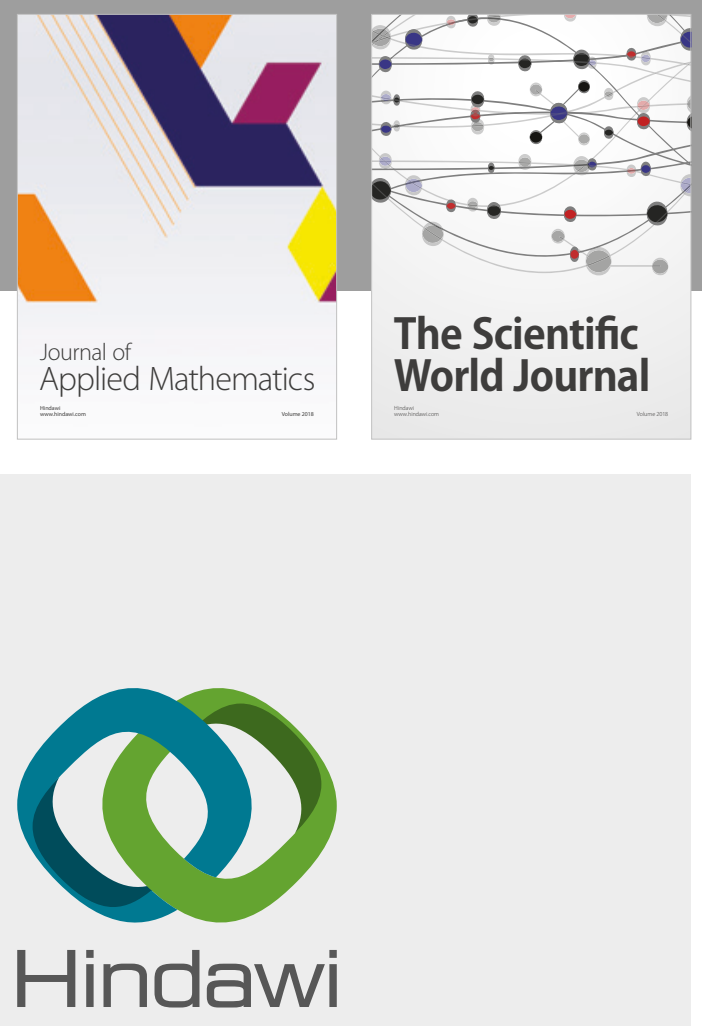

Submit your manuscripts at

www.hindawi.com

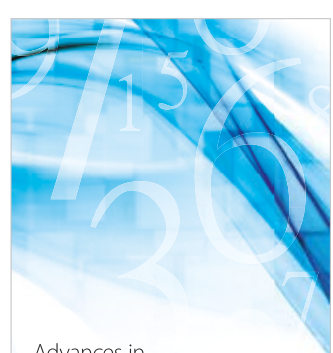

Advances in
Numerical Analysis
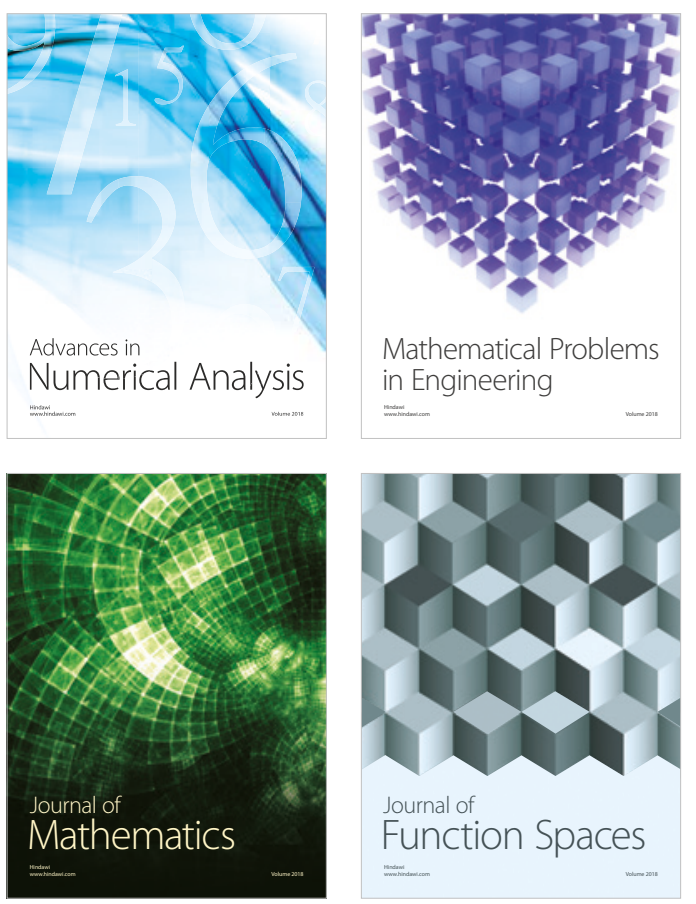

Mathematical Problems in Engineering

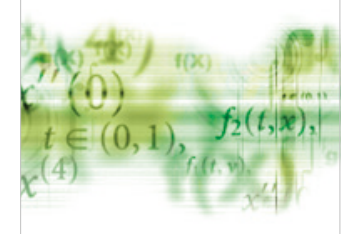

International Journal of

Differential Equations

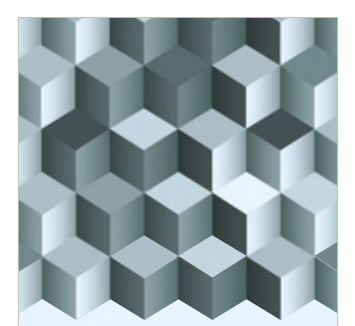

Journal of

Function Spaces

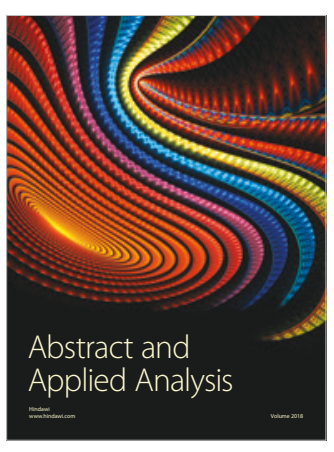

The Scientific

World Journal

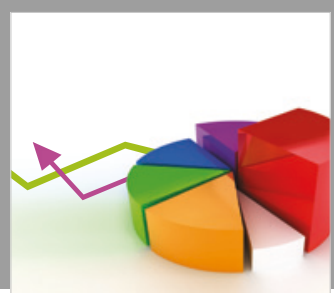

Journal of

Probability and Statistics
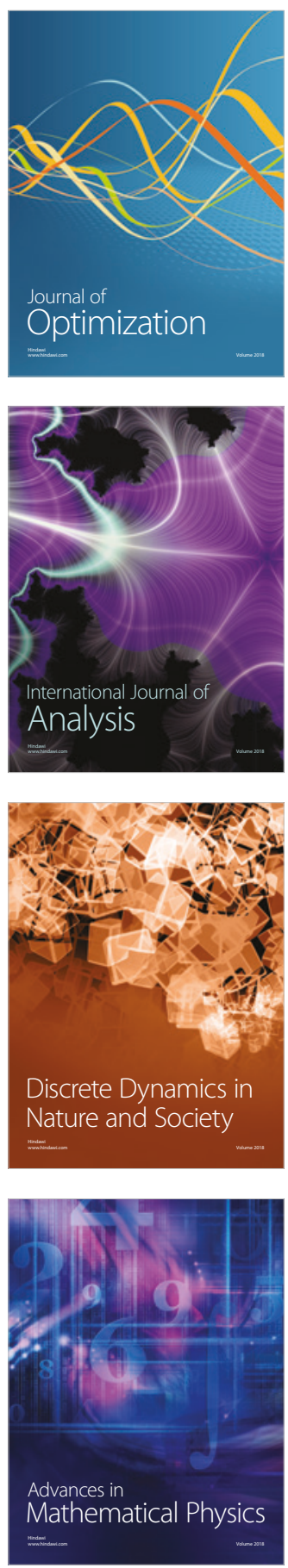